\title{
BMJ Open Association of lifestyle-related factors with circadian onset patterns of acute myocardial infarction: a prospective observational study in Japan
}

\author{
Ryuya Edahiro, ${ }^{1,2}$ Yasuhiko Sakata, ${ }^{1,3,4}$ Daisaku Nakatani, ${ }^{1}$ Shinichiro Suna, ${ }^{1}$ \\ Masaya Usami, ${ }^{1}$ Sen Matsumoto, ${ }^{1}$ Masahiko Hara, ${ }^{1}$ Tetsuhisa Kitamura, ${ }^{5}$ \\ Hiroshi Sato, ${ }^{6}$ Shizuya Yamashita, ${ }^{1,7}$ Shinsuke Nanto, ${ }^{1,3}$ Shungo Hikoso, ${ }^{1}$ \\ Yasushi Sakata, ${ }^{1}$ Masatsugu Hori, ${ }^{8}$ Toshimitsu Hamasaki, ${ }^{2}$ Issei Komuro, ${ }^{1,9}$ \\ on behalf of the OACIS Investigators
}

To cite: Edahiro R, Sakata $Y$, Nakatani D, et al. Association of lifestyle-related factors with circadian onset patterns of acute myocardial infarction: a prospective observational study in Japan. BMJ Open 2014;4:e005067. doi:10.1136/bmjopen-2014005067

- Prepublication history and additional material is available. To view please visit the journal (http://dx.doi.org/ 10.1136/bmjopen-2014005067).

Received 17 February 2014 Revised 26 April 2014 Accepted 2 May 2014

CrossMark

For numbered affiliations see end of article.

Correspondence to Dr Yasuhiko Sakata; sakatayk@cardio.med.tohoku. ac.jp

\section{ABSTRACT}

Objective: The onset of acute myocardial infarction (AMI) shows characteristic circadian variations involving a definite morning peak and a less-defined night-time peak. However, the factors influencing the circadian patterns of AMI onset and their influence on morning and night-time peaks have not been fully elucidated.

Design, setting and participants: An analysis of patients registered between 1998 and 2008 in the Osaka Acute Coronary Insufficiency Study, which is a prospective, multicentre observational study of patients with AMI in the Osaka region of Japan. The present study included 7755 consecutive patients with a known time of AMI onset.

Main outcomes and measures: A mixture of two von Mises distributions was used to examine whether a circadian pattern of AMI had uniform, unimodal or bimodal distribution, and the likelihood ratio test was then used to select the best circadian pattern among them. The hierarchical likelihood ratio test was used to identify factors affecting the circadian patterns of AMI onset. The Kaplan-Meier method was used to estimate survival curves of 1-year mortality according to AMI onset time.

Results: The overall population had a bimodal circadian pattern of AMI onset characterised by a high and sharp morning peak and a lower and less-defined night-time peak (bimodal $p<0.001$ ). Although several lifestyle-related factors had a statistically significant association with the circadian patterns of AMI onset, serum triglyceride levels had the most prominent association with the circadian patterns of AMI onset. Patients with triglyceride $\geq 150 \mathrm{mg} / \mathrm{dL}$ on admission had only one morning peak in the circadian pattern of AMI onset during weekdays, with no peaks detected on weekends, whereas all other subgroups had two peaks throughout the week.

Conclusions: The circadian pattern of AMI onset was characterised by bimodality. Notably, several lifestylerelated factors, particularly serum triglyceride levels, had a strong relation with the circadian pattern of AMI onset.

\section{Strengths and limitations of this study}

- We comprehensively analysed the circadian patterns of acute myocardial infarction (AMI) onset in a large, multicentre cohort of patients in relation to patient characteristics, lifestyle factors and the day of the week.

- A mixture of two von Mises distributions revealed that the circadian pattern of AMI onset exhibited bimodality.

- Several lifestyle-related factors were shown to be associated with the circadian patterns of AMI onset, depending on the day of the week. In particular, it was demonstrated that elevated serum triglyceride levels on admission accentuated morning peak of AMI onset during weekdays.

- Participants were limited to those who were hospitalised for AMI.

- Laboratory data were evaluated on admission.

Trial registration number: UMIN000004575.

\section{INTRODUCTION}

Onset patterns of acute myocardial infarction (AMI) exhibit circadian variation which is characterised by an increased frequency in the morning and a secondary peak incidence at night-time. ${ }^{1}$ Several studies have confirmed that AMI onset exhibits a bimodal circadian pattern, with peaks occurring in the morning ${ }^{2-4}$ and night-time hours. ${ }^{1}{ }^{4-7}$ However, it is not well understood what factors, particularly lifestylerelated factors, influence the circadian patterns of AMI. Moreover, although these patterns appear to vary according to the day of the week, ${ }^{8}$ it is unclear how the circadian patterns 
of AMI onset vary throughout the week, particularly, in association with socioeconomic factors.

As AMI and subsequent ischaemic heart failure is the leading cause of death in developed and developing countries, primary prevention of AMI is a major healthcare issue worldwide. Accordingly, identifying potential factors influencing the circadian pattern of AMI may help in the clinical management of patients to prevent the onset of AMI.

In the present study, we comprehensively analysed the circadian patterns of AMI onset in a large, multicentre cohort of patients in relation to patient characteristics, lifestyle factors and the day of the week.

\section{METHODS}

OACIS registry and study participants

The Organisation to Assess Strategies for Ischaemic Syndromes (OACIS) is a prospective, multicentre observational study collecting demographic, procedural, biological and outcome data as well as blood samples from patients with AMI hospitalised at 25 collaborating hospitals from the Osaka region of Japan (UMIN-Clinical Trial Registry ID: UMIN000004575; see online supplementary appendix). ${ }^{9}{ }^{10}$ A diagnosis of AMI was made if the patient fulfilled at least two of the following three criteria: (1) history of central chest pressure, pain or tightness lasting $30 \mathrm{~min}$, (2) typical ECG changes (ie, ST-segment elevation $\geq 0.1 \mathrm{mV}$ in one standard limb lead or two precordial leads, ST-segment depression $\geq 0.1 \mathrm{mV}$ in two leads, abnormal Q-waves or T-wave inversion in two leads) and (3) an increase in serum creatine kinase levels two times the upper normal limit in each hospital. All the collaborating hospitals were encouraged to enrol consecutive patients with AMI.

We prospectively collected data with the help of research cardiologists and trained research nurses using a specific reporting form, and the following variables were extracted from the OACIS registry database: age, gender, working status, body mass index, coronary risk factors (diabetes, hypertension, dyslipidaemia, smoking, drinking, previous MI, multivessel disease and collateral circulation), clinical presentation on admission (KILLIP classification, initial TIMI flow and ST-elevation MI (STEMI)), coronary angiography data, reperfusion therapy, laboratory data on admission (glycated haemoglobin (HbAlc), total cholesterol, low-density (LDL) and high-density lipoprotein (HDL) cholesterol, triglyceride and estimated glomerular filtration rate) and medications at discharge (RAS inhibitors, $\beta$-blocker, calcium channel blocker, statin, antiplatelet agent and diuretics). Diabetes mellitus was defined as fasting plasma glucose $\geq 126 \mathrm{mg} / \mathrm{dL}, \mathrm{HbAlc} \geq 6.5 \%$ or a history of antidiabetic therapy. Hypertension was defined as a history of systolic blood pressure $\geq 140 \mathrm{~mm} \mathrm{Hg}$, diastolic blood pressure $\geq 90 \mathrm{~mm} \mathrm{Hg}$ or antihypertensive therapy. Dyslipidaemia was defined as fasting total cholesterol $\geq 220 \mathrm{mg} / \mathrm{dL}$, LDL cholesterol $\geq 140 \mathrm{mg} / \mathrm{dL}$, HDL cholesterol $\leq 40 \mathrm{mg} / \mathrm{dL}$, fasting triglycerides $\geq 150 \mathrm{mg} / \mathrm{dL}$ or lipid-lowering therapy.
The study protocol has been approved by the ethics committee of each participating hospital. All in-hospital data were obtained after written informed consent had been received and were then transmitted to the data collection centre at the Department of Cardiovascular Medicine, Osaka University Graduate School of Medicine, Suita, Japan, for processing and analysis. The corresponding authors had full access to and validated all data in the study.

In the present study, we analysed 7755 patients with AMI whose time of AMI onset was definitely known among the 8603 consecutive patients registered in the OACIS registry between 1998 and 2008. Patients' baseline characteristics are presented in table 1.

\section{Statistical analysis}

Continuous variables were summarised as quartiles and were compared using the Wilcoxon rank-sum test for two-group comparisons, and the Kruskal-Wallis test for four-group comparisons. Categorical variables were presented as numbers and percentages, and were compared using the $\chi^{2}$ test. A mixture of two von Mises distributions was used to examine whether a circadian pattern of AMI onset had uniform (no peak), unimodal (one peak) or bimodal distribution (two peaks), and the likelihood ratio test was then used to select the best circadian pattern among them. ${ }^{11}$ In addition, the hierarchical likelihood ratio test was used to identify factors affecting the circadian patterns of AMI onset. The Kaplan-Meier method was used to estimate survival curves of 1-year mortality according to AMI onset time (morning (6:00-11:59), afternoon (12:00-17:59), evening (18:00-23:59) and night-time $(0: 00-5: 59))$. The log-rank test was used to compare survival curves between the groups, and the Cox proportional hazards regression model was used to estimate HRs and 95\% CIs. To reduce potential confounding effects due to patient background variability in the comparison between the afternoon-onset and other groups, a stratified Cox proportional hazards regression model was used, in which the potential confounding variables were included into the model as stratification factors. Cosinor analysis was used to estimate the amplitude of serum triglyceride (TG) levels on admission according to AMI onset time. Then, an F-test for the existence of a rhythm (amplitude) was used to examine whether the amplitude of serum TG levels on admission in patients with AMI had circadian variation or not. Statistical significance was set as $\mathrm{p}<0.05$. All statistical analyses were performed using an in-house validated Fortran program or SAS V.9.3 (SAS Institute Inc, Cary, North Carolina, USA).

\section{RESULTS}

Bimodal circadian patterns of AMI onset in the overall population

The daily patterns of AMI onset in our cohort of 7755 patients were first analysed using the likelihood ratio test (figure 1). In the overall population, AMI onset clearly 
Table 1 Demographics and clinical characteristics of the study population

\begin{tabular}{|c|c|}
\hline & $N=7755$ \\
\hline \multicolumn{2}{|l|}{ Patients } \\
\hline Age (years) & $66(57-74)$ \\
\hline Male (\%) & $5872(75.7)$ \\
\hline Job (\%) & 3364 (48.2) \\
\hline BMI $\left(\mathrm{kg} / \mathrm{m}^{2}\right)$ & $23.4(21.4-25.7)$ \\
\hline \multicolumn{2}{|l|}{ Cardiovascular risk factors } \\
\hline Smoker (\%) & 4865 (63.9) \\
\hline Drinker (\%) & 3321 (45.3) \\
\hline Diabetes (\%) & $2586(33.4)$ \\
\hline Hypertension (\%) & 4424 (58.9) \\
\hline Dyslipidaemia (\%) & $3259(44.1)$ \\
\hline Previous MI (\%) & 983 (13.0) \\
\hline Angina pectoris (\%) & $1737(23.4)$ \\
\hline Multivessel disease (\%) & $2790(38.4)$ \\
\hline Collateral circulation (\%) & $2576(35.7)$ \\
\hline \multicolumn{2}{|l|}{ Clinical presentation } \\
\hline Onset admission time $<24 \mathrm{~h}(\%)$ & $6804(89.1)$ \\
\hline KILLIP $\geq \mathrm{II}(\%)$ & $1331(18.0)$ \\
\hline Initial TIMI $\leq$ II (\%) & $4759(68.4)$ \\
\hline STEMI $(\%)$ & 6567 (86.0) \\
\hline \multicolumn{2}{|l|}{ Laboratory data on admission } \\
\hline Blood glucose level (mg/dL) & 152 (122-209) \\
\hline HDL cholesterol (mg/dL) & $44(37-53)$ \\
\hline LDL cholesterol (mg/dL) & $121(99-147)$ \\
\hline Triglycerides (mg/dL) & $92(58-142)$ \\
\hline HbA1c (\%) & $5.9(5.5-6.9)$ \\
\hline Peak CK (IU/L) & $2147(1069-4006)$ \\
\hline eGFR $\left(\mathrm{mL} / \mathrm{min} / 1.73 \mathrm{~m}^{2}\right)$ & $64.5(49.2-80.9)$ \\
\hline \multicolumn{2}{|l|}{ Localisation of MI } \\
\hline LAD & 3050 (41.7) \\
\hline $\mathrm{RCA}$ & 2447 (33.4) \\
\hline LCX & 998 (13.6) \\
\hline LMT & 164 (2.2) \\
\hline
\end{tabular}

Categorical variables are presented as number (\%), and continuous variables are presented as quartile. Laboratory data were measured on admission. Smoker was defined as a patient with a smoking history, and drinker was defined as an active drinker. Number (\%) of localisation of MI was calculated out of 7319 patients who underwent coronary angiography.

$\mathrm{BMI}$, body mass index; CK, creatine kinase; eGFR, estimated glomerular filtration rate; $\mathrm{HbA1c}$, glycated haemoglobin; $\mathrm{HDL}$, high-density lipoprotein; LAD, left anterior descending artery; LCX, left circumflex artery; LDL, low-density lipoprotein; LMT, left main trunk; MI, myocardial infarction; RCA, right coronary artery; STEMI, ST-elevation myocardial infarction.

exhibited a circadian pattern consisting of two peaks (bimodal: $\mathrm{p}<0.001)$ : a primary peak at 9:01 $(95 \%$ CI 8:53 to 9:08) and a secondary peak at 20:11 (95\% CI $19: 48$ to $20: 34)$. The primary peak was more clearly defined than the secondary peak in the circular and columnar histograms (figure 1A, B, respectively).

The likelihood ratio test analysis revealed that the peak time of AMI onset varied according to the day of the week (figure 2). For example, the primary peak onset time was earliest on Monday (8:24 (95\% CI 8:04 to 8:44)) and latest on Sunday (9:44 (95\% CI 9:22 to 10:06)). On Tuesday, patients exhibited a circadian
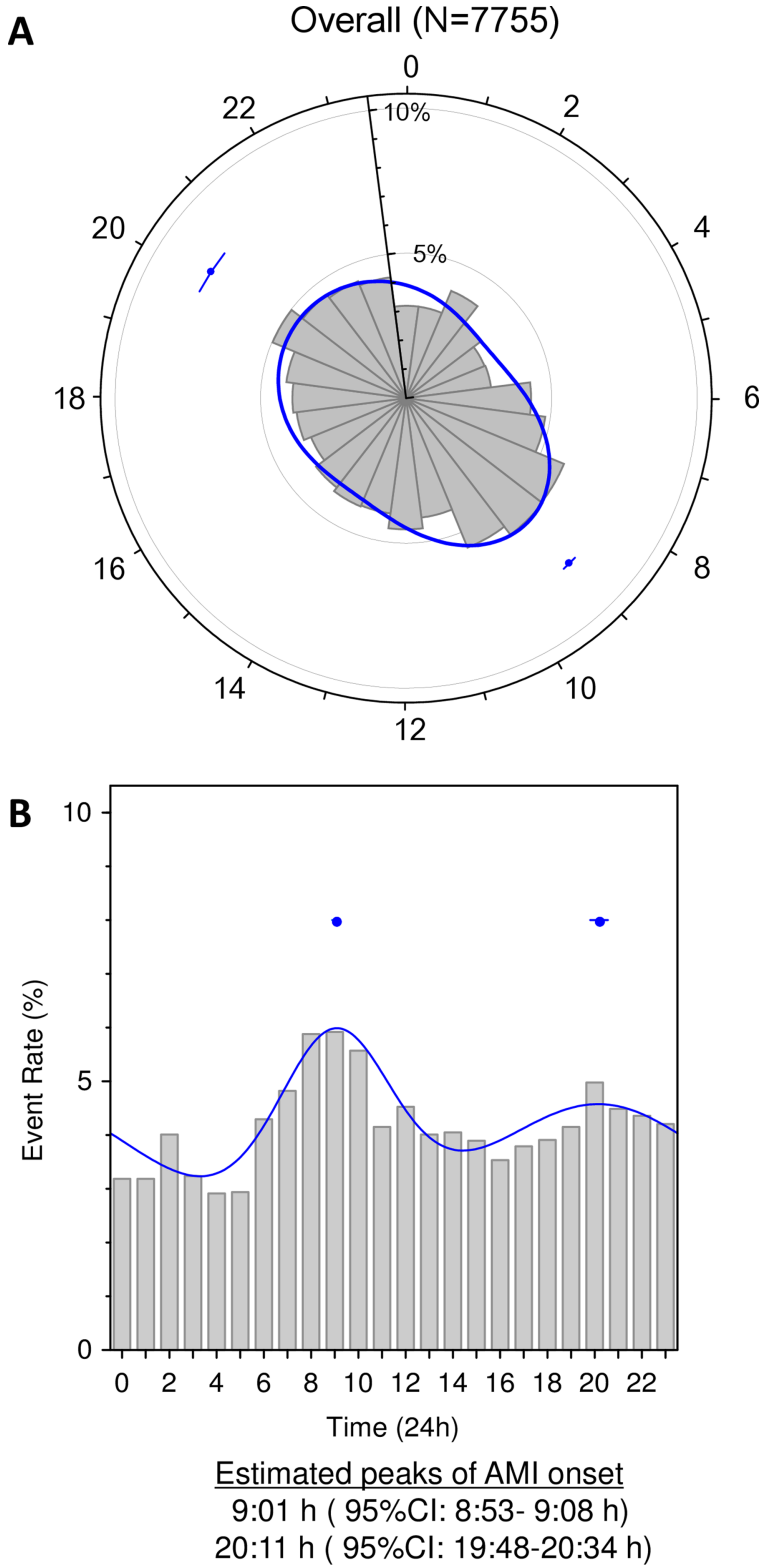

Figure 1 Circadian pattern of acute myocardial infarction (AMI) onset in the overall population. A circadian pattern of AMI onset in the overall population was clearly observed in a circular plot (A) and histogram (B). The solid line corresponds to the fitted von Mises distribution, and the dots with error bars are the estimated peak onset times and $95 \% \mathrm{Cls}$, respectively.

pattern of AMI onset characterised by late primary (9:28 (95\% CI 9:06 to 9:51)) and secondary peak onset times (21:13 (95\% CI 20:40 to 21:46)), whereas earlier peak onset times (8:43 (95\% CI 8:15 to 9:10) and 19:09 (95\% CI 18:23 to 19:55)) were detected on Thursday. Notably, the evening peak was higher and sharper than the morning peak on Saturday (figure 2).

\section{Factors affecting the circadian patterns of AMI onset}

The hierarchical likelihood ratio analysis revealed that serum TG levels on admission, smoking, age, drinking, blood glucose levels on admission, gender and working 
Monday $(\mathrm{N}=1133)$

Tuesday $(\mathrm{N}=1129)$

Wednesday $(\mathrm{N}=1096)$

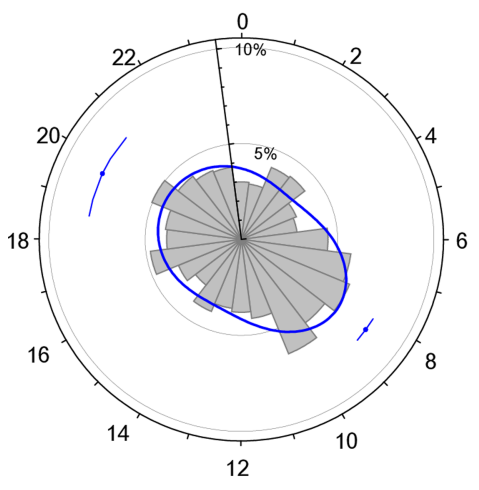

Bimodal: $p<0.001^{*}$

$8: 24 \mathrm{~h}(95 \% \mathrm{Cl}: 8: 04-8: 44 \mathrm{~h})$

19:40 h (95\%Cl: $18: 35-20: 46$ h)

Thursday $(\mathrm{N}=1086)$

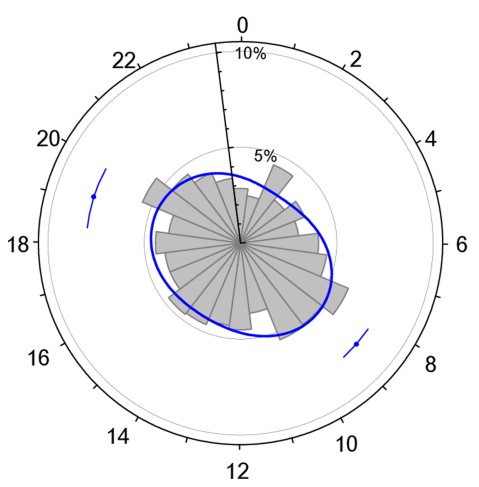

Bimodal: $p<0.001$

$8: 43 \mathrm{~h}(95 \% \mathrm{Cl}: 8: 15$ - 9:10 h) 19:09 h (95\%Cl: $18: 23-19: 55$ h)

\section{Sunday $(N=1061)$}

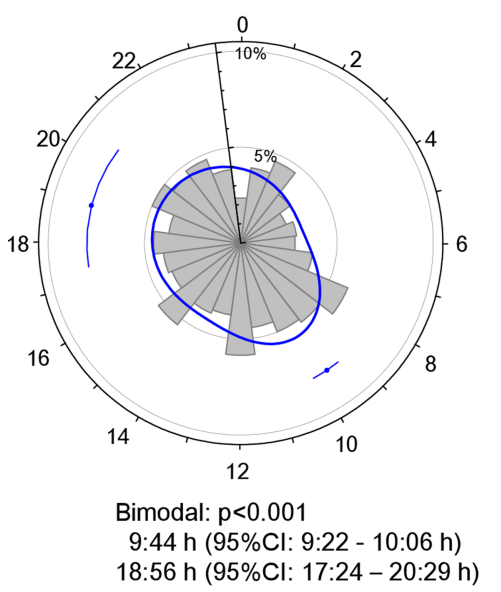

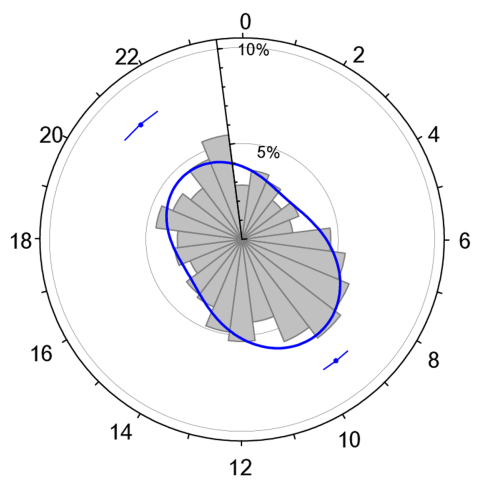

Bimodal: $p<0.001$

9:28 h (95\%Cl: 9:06 - 9:51 h)

21:13 h (95\%Cl: $20: 40-21: 46$ h)

Friday $(\mathrm{N}=1165)$

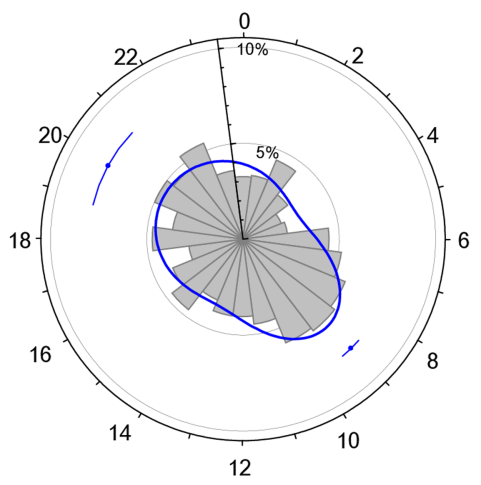

Bimodal: $p<0.001$

9:02 h (95\%Cl: 8:45 - 9:19 h)

19:55 h (95\%Cl: 18:53 - 20:56 h)

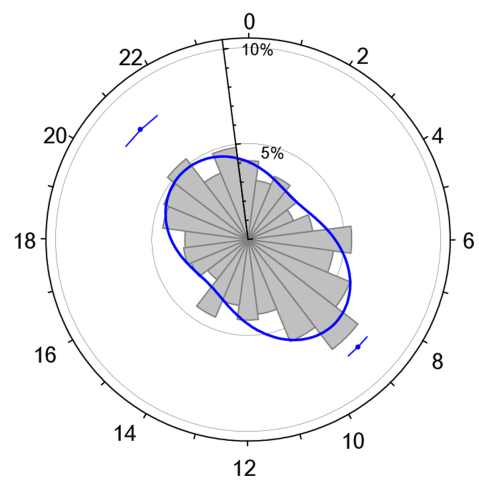

Bimodal: $p<0.001$

$8: 55 \mathrm{~h}(95 \% \mathrm{Cl}: 8: 34$ - 9:15 h)

21:01 h (95\%Cl: $20: 28-21: 34$ h)

Saturday $(\mathrm{N}=1085)$

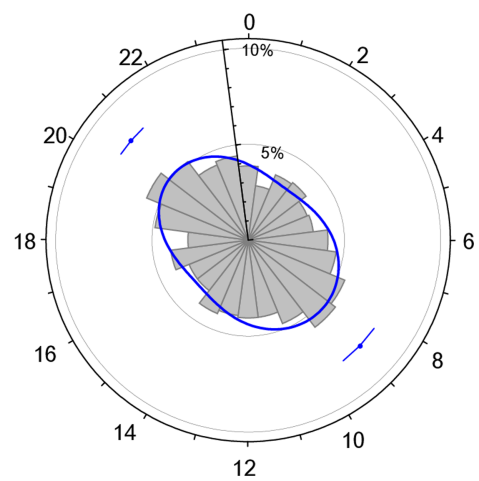

Bimodal: $p<0.001$

$8: 53 \mathrm{~h}(95 \% \mathrm{Cl}: 8: 20$ - 9:27 h)

20:41 h (95\%Cl: 20:16 - 21:07 h)

Figure 2 Circadian pattern of acute myocardial infarction (AMI) onset according to the day of the week. Circadian patterns of AMI onset based on the day of the week are shown. The estimated peak onset time and $95 \%$ Cls are shown below each circular plot. *p Values from the likelihood ratio test to examine whether the circadian pattern of AMI onset was uniform, unimodal or bimodal.

status had a statistically significant association with the circadian pattern of AMI onset, whereas several other known risk factors for AMI, including HDL and LDL cholesterol, HbA1c, hypertension, diabetes and dyslipidaemia were not related to the observed patterns (figure 3, supplementary table 1).

Among the positively associated factors, serum TG levels on admission had the greatest association with the 


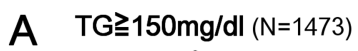

$\mathrm{TG}<150 \mathrm{mg} / \mathrm{dl}(\mathrm{N}=5055)$
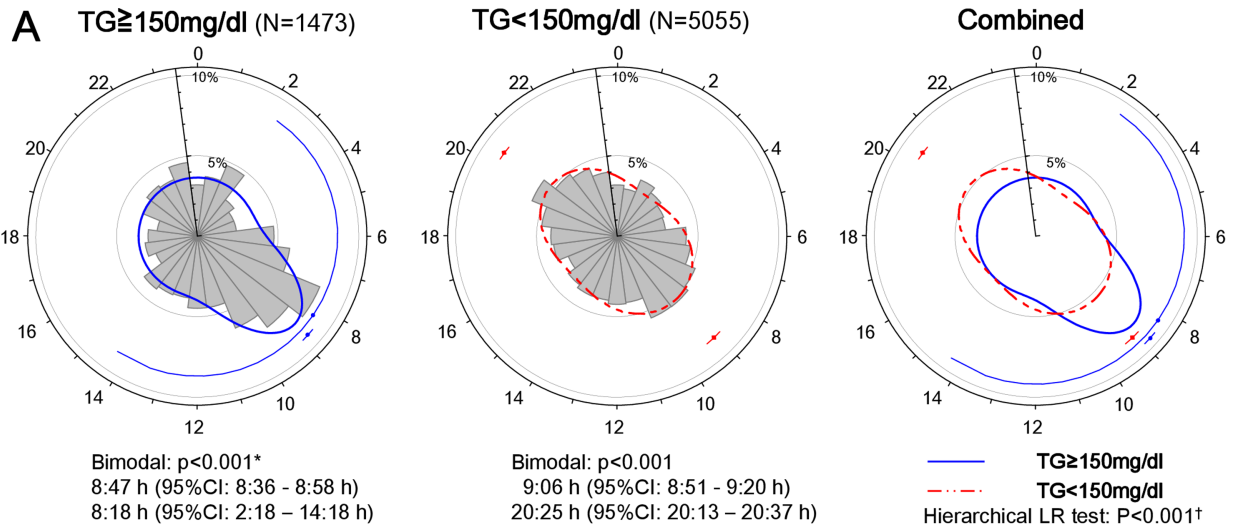

Bimodal: $p<0.001$

Bimodal: $p<0.001^{*}$

$8: 47 \mathrm{~h}(95 \% \mathrm{Cl}: 8: 36$ - 8:58 h)

$20.25 \mathrm{~h}$

Hierarchical LR test: $P<0.001$
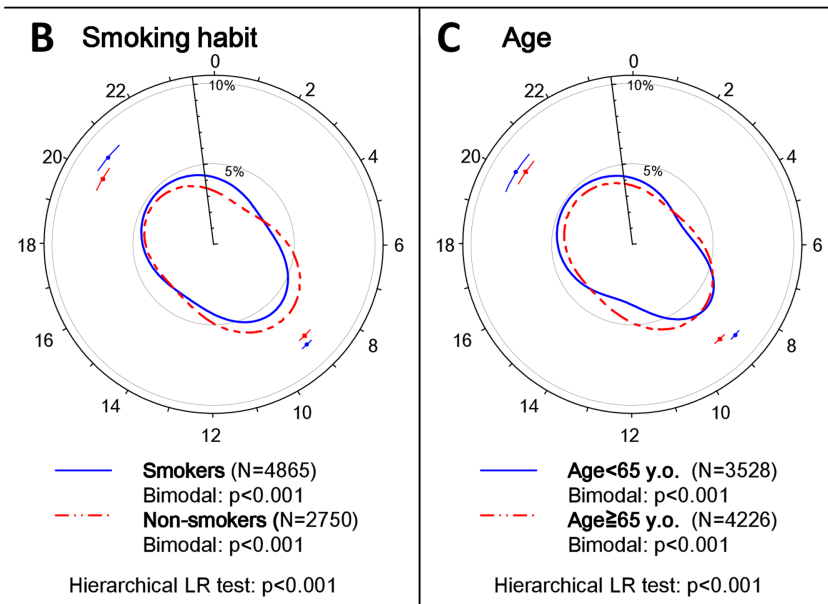

D Drinking habit
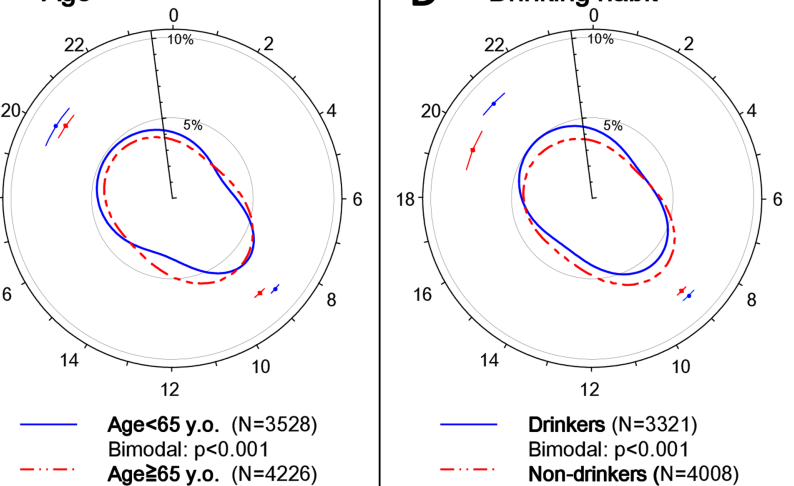

Age $\geq 65$ y.o. $(\mathrm{N}=42$

Bimodal: $p<0.001$

- Drinkers $(\mathrm{N}=3321)$

-... Non-drinkers $(\mathrm{N}=4008)$

Bimodal: $p<0.001$

E Blood glucose

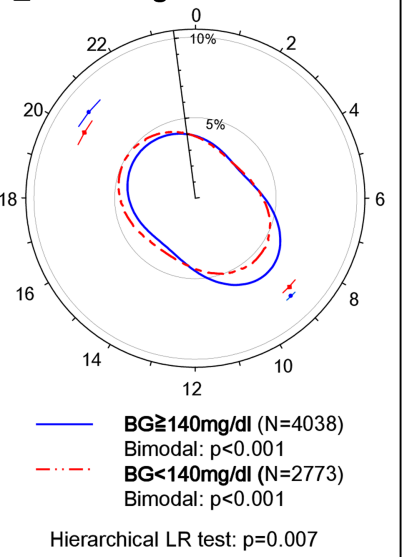

Hierarchical LR test: $p<0.001$

Hierarchical LR test: $p<0.001$
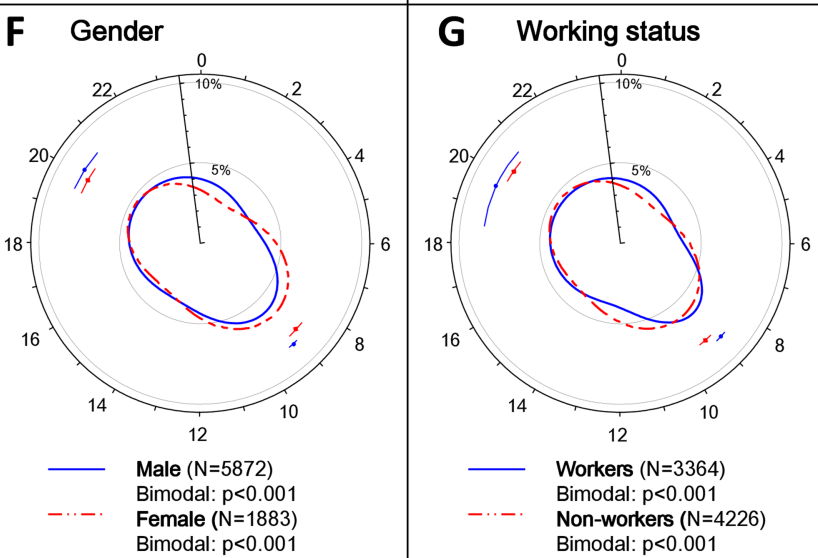

Hierarchical LR test: $p=0.010$

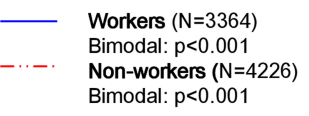

Hierarchical LR test: $p=0.048$

Figure 3 Circadian pattern of acute myocardial infarction (AMI) onset based on lifestyle-related factors. (A) Circular plots of the circadian pattern of AMI onset in the subpopulation with triglyceride (TG) levels $\geq 150$ and $<150 \mathrm{mg} / \mathrm{dL}$, and the circular plot of the corresponding fitted von Mises distributions for each subgroup are shown. (B-M) Circular plots of the fitted von Mises distributions of each subgroup based on smoking habit, age, drinking habit, blood glucose levels, gender and working status, low-density lipoprotein (LDL) levels, high-density lipoprotein (HDL) levels, glycated haemoglobin (HbA1c) levels, hypertension, diabetes and dyslipidaemia. ${ }^{*} p$ Values from the likelihood ratio (LR) test to examine whether the circadian pattern of AMI onset was uniform, unimodal or bimodal in each subgroup. $\mathrm{tp}$ Values from the hierarchical LR test to examine whether each factor affected the circadian pattern of AMI onset.

circadian pattern of AMI onset. Although the likelihood ratio test demonstrated that patients with admission serum TG levels of $\geq 150 \mathrm{mg} / \mathrm{dL}(\mathrm{N}=1473$ ) had two characteristic peaks during the day, the peak pattern clearly differed from the other subpopulation groups. In patients with admission serum TG levels of $\geq 150 \mathrm{mg} / \mathrm{dL}$, both peaks occurred in the morning and nearly overlapped (8:18 and 8:47; figure 3A). Therefore, the subpopulation with admission TG levels $\geq 150 \mathrm{mg} / \mathrm{dL}$ was considered to have a high frequency of AMI onset only in the morning.

The baseline characteristics and laboratory data of patients with serum TG levels of $\geq 150$ and $<150 \mathrm{mg} / \mathrm{dL}$ 


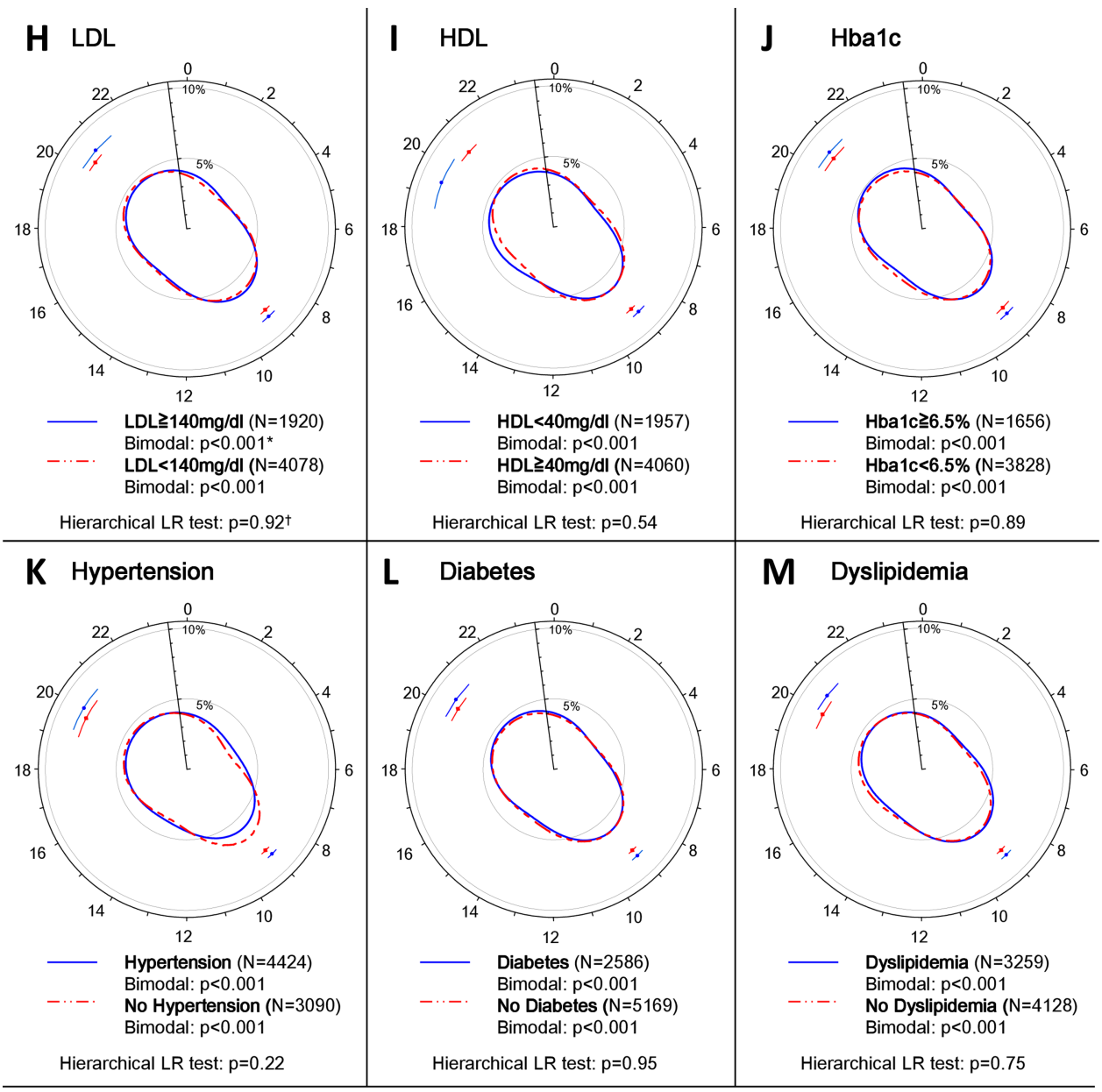

Figure 3 Continued.

on admission are shown in supplementary table 2. In the subpopulation with higher TG levels, the circadian patterns of AMI onset were characterised by a large, sharp peak in the morning from Monday to Friday, but no peaks were detected on Saturday and Sunday (bimodal: $p=0.32$ and 0.133 , respectively; supplementary figure 1). In contrast, patients with admission serum TG levels of $<150 \mathrm{mg} / \mathrm{dL}(\mathrm{N}=5055)$ had onset peaks that occurred in the morning and evening consistently throughout the week (supplementary figure 1).

A likelihood ratio test demonstrated that all other subpopulations had two AMI onset peaks during the day: one in the morning and the other in the evening (figure 3 , supplementary table 1 ). The subpopulations that were grouped according to smoking habit, age $<65$ years, male gender and active employment had a circadian pattern of AMI onset with a sharper primary peak and a less-defined sharp secondary peak compared with the other subpopulations (figure 3B, C, F, G, supplementary table 1), although the peak heights were similar between the subpopulations, with the exception of the smoker/non-smoker subpopulations. The primary AMI onset peak in the subpopulation of smokers was higher than that among non-smokers, whereas the secondary peaks were similar. Drinkers had a circadian pattern of AMI onset that was characterised by a lower and less sharp peak in the morning and a higher, sharper and later peak in the evening (9:00 (95\% CI 8:48 to $9: 13,20: 54$ (95\% CI 20:29 to 21:20)) compared with non-drinkers (9:03 (95\% CI 8:53 to 9:14), 19:27 (95\% CI 18:50 to 20:04); figure 3D, supplementary table 1). The subpopulation with admission blood glucose $\geq 140 \mathrm{mg} / \mathrm{dL}$ exhibited a circadian pattern of AMI onset with a higher and sharper primary peak and a less-defined secondary peak compared with the subpopulation of patients with AMI with blood glucose $<140 \mathrm{mg} / \mathrm{dL}$ on admission (figure $3 \mathrm{E}$ ).

\section{One-year mortality according to onset time of AMI}

One-year mortality was compared among four patient subpopulations that were grouped according to the time range of AMI onset. The baseline characteristics and laboratory data for the four groups are presented in supplementary table 3 . A total of 753 deaths were recorded during a median follow-up period of 365 days. The Kaplan-Meier survival analysis demonstrated that the afternoon-onset (12:00-17:59) group had worse 1-year mortality than the other three groups (log-rank test, $\mathrm{p}=0.032$; figure $4 \mathrm{~A})$. In the subgroup of patients with STEMI, the result was similar (log-rank test, $\mathrm{p}=0.007)$. The 


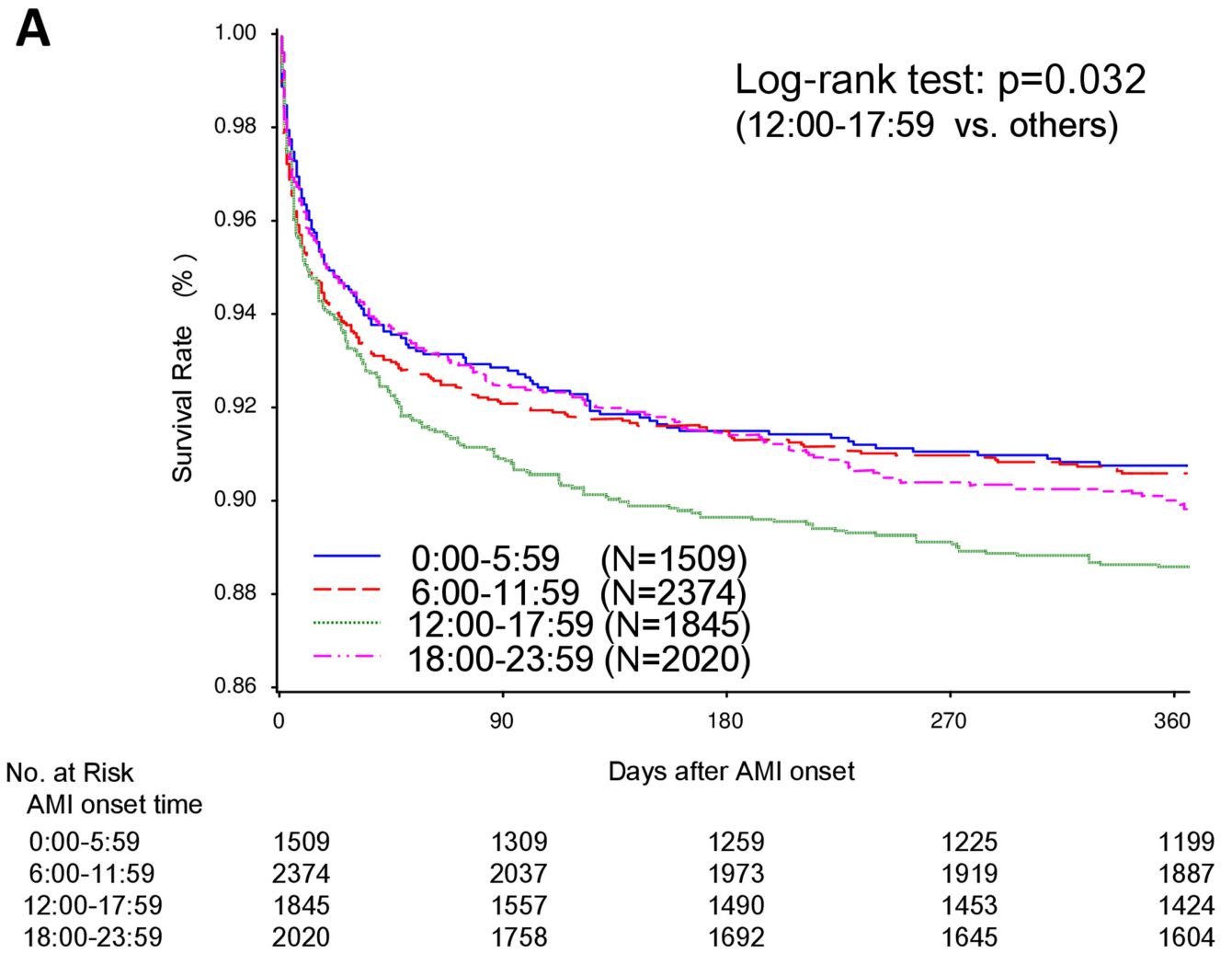

B

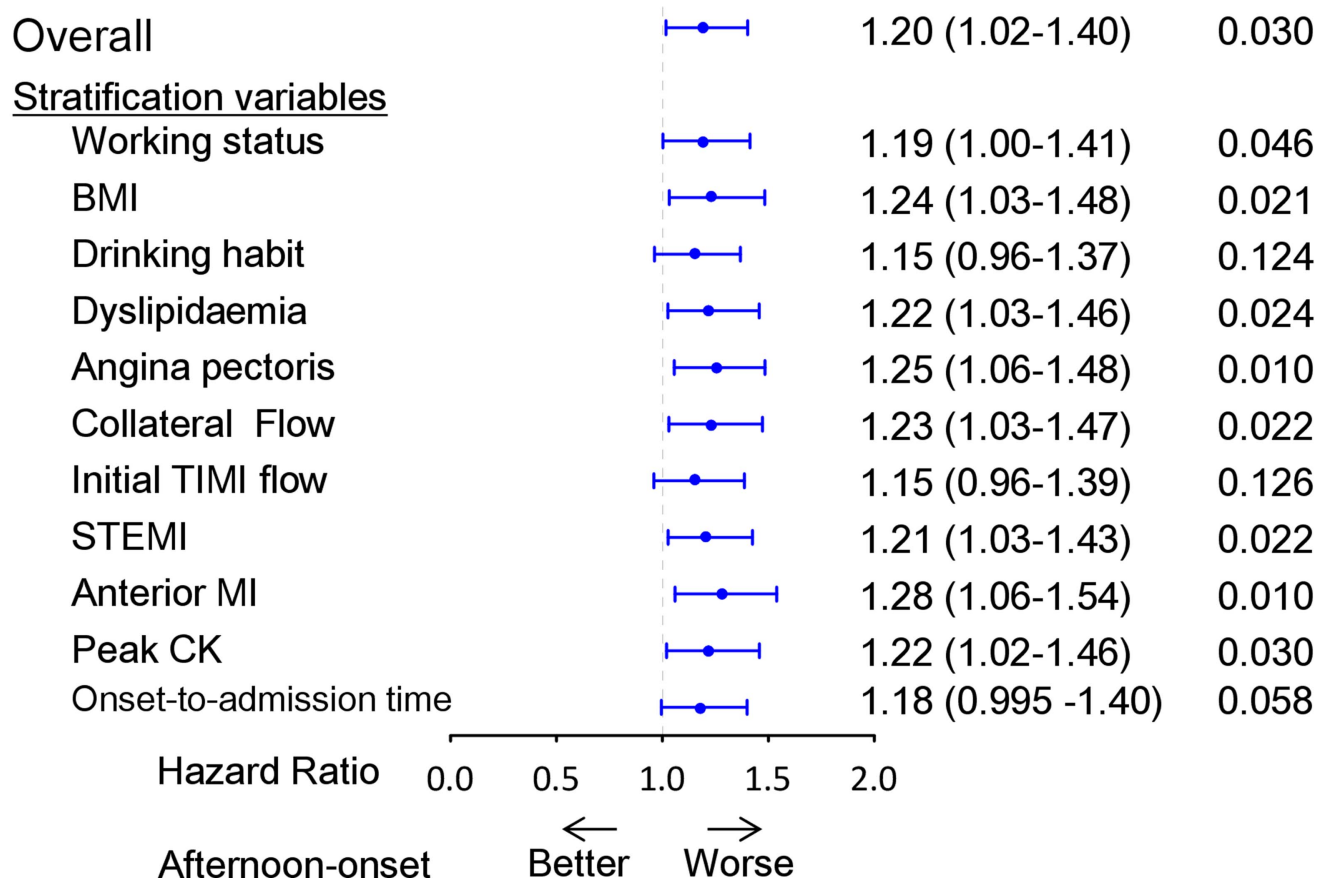

Figure 4 One-year mortality according to the onset time of AMI onset. (A) One-year mortality among the four subgroups based on AMl onset time. (B) HRs for 1-year mortality in the afternoon-onset group versus the other three onset time groups. The Kaplan-Meier survival curves of 1-year mortality among the four AMI onset time subgroups (A). A p value from the log-rank test was used to examine difference in the Kaplan-Meier curves. The $\mathrm{HR}$ and $95 \% \mathrm{Cl}$, and $\mathrm{p}$ value for the overall population was calculated using univariable Cox regression analysis. The $\mathrm{HRs}$ and $95 \% \mathrm{Cls}$, and $\mathrm{p}$ values for the individual potential confounding variables were calculated using stratified Cox regression analysis, in which the variables were included into the model as stratification factors (B). AMI, acute myocardial infarction; BMI, body mass index; CK, creatine kinase; STEMI, ST-elevation MI. 
univariable Cox regression analysis revealed that the $\mathrm{HR}$ of 1-year mortality in the afternoon-onset group as compared with the other three groups was 1.20 (95\% CI 1.02 to 1.40, $\mathrm{p}=0.030$, figure $4 \mathrm{~B}$ ). This result did not generally change after stratification with potential confounding factors that showed a different trend between the afternoon-onset group and the other three groups (figure 4B).

\section{DISCUSSION}

In the present study, we confirmed that AMI onset exhibits a circadian pattern characterised by bimodality, with a definite morning peak and a less-defined evening peak. Notably, several lifestyle-related factors were associated with variation in the circadian pattern of AMI onset. In particular, serum TG levels on admission for AMI were associated with a unique pattern of AMI onset that is characterised by augmented unimodal peaks on weekday mornings, suggesting that an individual's lifestyle may affect the onset pattern of AMI.

\section{Bimodal pattern of AMI onset: morning and night-time peaks}

AMI onset in our large patient cohort generally followed a circadian pattern that was characterised by a high and sharp morning peak and a lower and less-defined sharp night-time peak (figure 1), a finding that is consistent with the results of previous investigations. ${ }^{1-7}$ Interestingly, the time of two peaks shifted in a synchronous fashion during weekdays; the secondary peaks generally occurred around 11-12 $\mathrm{h}$ after the morning peaks on Monday through Friday (figure 2). For example, AMI onset exhibited early morning and night-time peaks on Monday and Thursday, whereas that on Tuesday exhibited late morning and night-time peaks. Although this finding is partly consistent with the observation of Peters $e t a l,{ }^{5}$ who reported that a secondary peak in AMI onset occurs 11$12 \mathrm{~h}$ after waking, the present study first demonstrated that this synchrony was present on weekdays, but absent on weekends.

Several physiological processes are considered to contribute to the bimodal pattern of AMI onset. For example, Stergiou et $\mathrm{al}^{12}$ demonstrated that the two-peak diurnal variation in stroke onset occurs in parallel with variation in blood pressure, pulse rate and physical activity. Thus, the bimodality of blood pressure and heart rate $^{1314}$ is the most likely explanation for the circadian patterns of AMI onset observed in the present study. A greater morning surge of blood pressure and heart rate $^{13}$ may explain why the night-time peak of AMI onset was lower and less-defined than the morning peak. In addition, increased blood viscosity ${ }^{15}$ and thrombogenicity due to morning hypercoagulability ${ }^{16}$ and hypofibrinolysis $^{17}$ also likely increased the frequency of AMI onset in the morning. It is also possible that external factors, such as physical exertion and mental stress, could be triggers for the morning onset of AMI. ${ }^{18}$ In the present study, the younger ( $<65$ years old), working, male and smoker subpopulations had a sharp morning peak of AMI onset compared with the elderly, nonworking, female and non-smoking subpopulations (figure 3B, C, F, G). The sharpness of the morning peak might be related to increased susceptibility to physical and mental stress in these subpopulations, when they are more likely to start activities or go to work soon after waking up. Similarly, the sharp and early morning peak of AMI onset that was detected on Monday may be due to the increased physical and mental stress that is associated with the first morning of the week (figure 2). We also found that the morning peak occurred latest on Sunday (figure 2). Together, these findings strongly suggest that mental and physical activity and/or stress may act as a trigger for the morning onset of AMI.

Although many reports have examined the primary peak of AMI onset, relatively little attention has been paid to the secondary peak. We demonstrated that drinkers had a higher, sharper and later night-time peak of AMI onset than non-drinkers (figure 3D). Moreover, the night-time peak on Saturday was the highest and sharpest among the 7 days of the week (figure 2). This observation may be explained by the fact that people might likely consume alcohol and engage in social activities on Saturday night in Japan. Thus, these evening activities can result in increased sympathetic nerve activity and therefore may have contributed to the increased frequency of AMI onset at night. Taken together, our findings suggest that the morning and night-time peaks of AMI onset are influenced by physiological and socioeconomic factors.

\section{Associations of lifestyle-related factors with the circadian patterns of AMI onset}

Many previous studies on the circadian pattern of AMI onset considered gender, age, working status as potential factors affecting the circadian patterns of AMI onset. ${ }^{1}{ }^{4-6}$ We additionally incorporated laboratory data, disease and other socioeconomic factors into our analyses and found that several lifestyle-related factors, including admission serum TG and blood glucose levels, age, gender, working status and smoking and drinking habits had statistically significant associations with the circadian pattern of AMI onset. Among these factors, elevated serum TG levels $(\geq 150 \mathrm{mg} / \mathrm{dL})$ on admission had the largest associations with the circadian patterns of AMI onset, while the amplitude of serum TG levels on admission in patients with AMI did not have circadian variation $(\mathrm{p}=0.52$; supplementary figure 2).

There are several evidences to support our findings. First, fasting hypertriglycaemia and postprandial hyperlipidaemia, which is characterised by postprandial accumulation of TG-rich lipoproteins and their partially hydrolysed products, are closely related to the development of atherosclerotic cardiovascular diseases. ${ }^{19-21}$ Several studies have also reported that elevated serum TG levels are associated with an increased risk of MI. ${ }^{22}{ }^{23}$ Hypertriglycaemia is associated with increased 
thrombogenicity, ${ }^{24}{ }^{25}$ which is reportedly associated with increased plasminogen activator inhibitor-1 (PAI-1) ${ }^{26-28}$ and factor VII coagulant activities, ${ }^{29}{ }^{30}$ and viscosity. ${ }^{31}$ These three factors have also been reported to affect the development of MI. ${ }^{32-34}$ Moreover, hypertriglyceridaemia is also related to endothelium dysfunction, ${ }^{35} 36$ which contributes to the pathogenesis of coronary artery disease. ${ }^{37}$ In healthy participants, serum TG levels also exhibit circadian variation with a peak around 3:00. ${ }^{38}$ Thus, it is conceivable that patients with hypertriglycaemia have further augmented TG levels and are therefore exposed to increased thrombogenicity and endothelium dysfunction in the early morning hours before dawn, which may explain the accentuated morning peak of AMI onset in patients with admission TG $\geq 150 \mathrm{mg} / \mathrm{dL}$. Finally, it is reported that high plasma PAI-1 levels and excessive surges in morning blood pressure are independently and additively associated with increased risk of stroke in older patients with hypertension. ${ }^{39}$ Thus, these lines of evidence strongly support our observation of a higher morning risk of AMI onset in the subpopulation with admission hypertriglyceridaemia.

\section{Altered circadian patterns of AMI onset in patients with increased TG levels on admission}

To the best of our knowledge, this is the first study to demonstrate an association between admission serum TG levels and the circadian patterns of AMI onset, as characterised by a lack of an evening peak in AMI onset in the subgroup of serum TG levels on admission $\geq 150 \mathrm{mg} / \mathrm{dL}$ compared with all other subgroups (figure 3). While LDL/HDL levels are considered to be closely associated with the development of atherosclerosis, LDL/HDL levels were not associated with onset patterns of AMI in the present study. Although the precise mechanisms for altered circadian patterns of AMI onset in patients with increased admission serum TG levels are unclear, increased serum TG might have influenced peripheral clocks residing in various tissues throughout the body, disrupting the circadian patterns of AMI onset. Indeed, recent studies have shown that energy metabolism is an important modulator of peripheral circadian clock in cardiovascular tissues. ${ }^{40} 41$

Our subpopulation analyses also revealed that the circadian patterns of AMI onset in patients with admission TG levels of $\geq 150 \mathrm{mg} / \mathrm{dL}$ had a sharp morning peak during weekdays, whereas no such peak was detected on Saturday or Sunday. This observation strongly suggests that increased thrombogenicity and endothelium dysfunction was a factor, but not the trigger, for the morning onset of AMI in our study cohort. Thus, it is conceivable that the accentuated morning peak of AMI onset in patients with admission TG $\geq 150 \mathrm{mg} / \mathrm{dL}$ may be due to the combination of the following three factors: (1) increased hypercoagulability, hypofibrinolysis, viscosity and endothelium dysfunction resulting from elevated serum TG levels, (2) increased risk of a morning surge of blood pressure and heart rate and (3) mental and physical stress.

\section{One-year mortality according to AMI onset time}

The association between AMI onset time and mortality is controversial. For example, Manfredini $e t a t^{42}$ reported that patients with a morning onset of AMI are characterised by higher fatal outcome, independent of site and size of infarction, while Bae $e t a t^{43}$ reported that patients with an evening-onset AMI had the worst 1-year mortality in association with poor baseline clinical characteristics. On the other hand, Holmes et at ${ }^{44}$ observed no significant association between the circadian patterns of onset time and in-hospital mortality in patients with STEMI after adjusting for clinical risk factors.

In the present study, patients with an afternoon onset of AMI had the worst 1-year mortality (figure 4A). However, the baseline clinical characteristics were comparable among the four onset time groups in our study cohort. Indeed, stratification for potential confounding variables did not generally change the results, suggesting that the increased prognostic risk of AMI in the afternoon-onset group was not simply explained by differences in baseline characteristics in the present study (figure 4B). Anyway, a patient's background and physiological circadian rhythms might complexly interact with each other and affect mortality after AMI, which could lead to these different results among the studies and difficulty in interpreting the results. Further investigations are required to clarify the association of mortality after AMI and onset time.

\section{Limitations}

A few limitations of the present study warrant mention. First, this was an analysis of a prospective observational study and the results may have therefore been influenced by potential confounding factors, even after adjustment for baseline clinical and angiographic characteristics. Thus, caution is needed when interpreting the data and making generalisations to other cohorts. Second, the laboratory findings, including serum TG levels, were evaluated on admission. Therefore, we could not exclude the influence of food consumption and circadian variation of several factors, particularly serum TG levels, making interpretation of the data difficult. However, our results also demonstrated that serum TG levels were not likely the final trigger for AMI onset, as patients with $\mathrm{TG} \geq 150 \mathrm{mg} / \mathrm{dL}$ on admission did not exhibit a morning peak of AMI onset on the weekend. In patients with hypertriglycaemia, hypercoagulability, hypofibrinolysis, viscosity and endothelium dysfunction are generally increased during the early morning hours before dawn, ${ }^{26-31} 353638$ resulting in enhanced susceptibility to AMI onset. Thus, under such conditions, it is conceivable that increased sympathetic activity, which was further enhanced in association with mental, physical and/or other factors, could be the final trigger for AMI onset on weekday mornings in patients with $\mathrm{TG} \geq 150 \mathrm{mg} / \mathrm{dL}$ on admission. Based 
on these findings, the influence of meal intake and circadian variation of serum TG levels on the morning peak of AMI onset in the population with TG $\geq 150 \mathrm{mg}$ / $\mathrm{dL}$ may be minimal, if not negligible.

\section{CONCLUSIONS}

In our large cohort of consecutive patients with AMI, the circadian pattern of AMI onset exhibited bimodality and was shown to be associated with several lifestyle-related factors. Among these factors, increased serum TG levels on admission had the most marked association with circadian variation, which was characterised by an increased morning risk of AMI onset during weekdays in this subpopulation. Our findings may help to identify the underlying triggers and substrates of AMI onset and help suggest preventive measures of AMI. However, caution is warranted to interpret our results and confirmation in other cohorts is required.

\section{Author affiliations}

${ }^{1}$ Department of Cardiovascular Medicine, Osaka University Graduate School of Medicine, Suita, Japan

${ }^{2}$ Department of Biomedical Statistics, Osaka University Graduate School of Medicine, Suita, Japan

${ }^{3}$ Department of Advanced Cardiovascular Therapeutics, Osaka University Graduate School of Medicine, Suita, Japan

${ }^{4}$ Department of Cardiovascular Medicine, Tohoku University Graduate School of Medicine, Sendai, Japan

${ }^{5}$ Department of Social and Environmental Medicine, Osaka University Graduate School of Medicine, Suita, Japan

${ }^{6}$ School of Human Welfare Studies Health Care Center and Clinic, Kwansei Gakuin University, Nishinomiya, Japan

${ }^{7}$ Department of Community Medicine, Osaka University Graduate School of Medicine, Suita, Japan

${ }^{8}$ Osaka Prefectural Hospital Organization, Osaka Medical Center for Cancer and Cardiovascular Diseases, Osaka, Japan

${ }^{9}$ Department of Cardiovascular Medicine, University of Tokyo Graduate School of Medicine, Tokyo, Japan

Acknowledgements The authors would like to thank Mariko Kishida, Rie Nagai, Nanase Muraoka, Hiroko Takemori, Akiko Yamagishi, Kumiko Miyoshi, Chizuru Hamaguchi, Hiroko Machida, Mariko Yoneda, Nagisa Yoshioka, Mayuko Tomatsu, Kyoko Tatsumi, Tomoko Mizuoka, Shigemi Kohara, Junko Tsugawa, Junko Isotani, Sachiko Ashibe, Satomi Kishimoto, Mayumi Maeda, and all other OACIS research coordinators and nurses for their excellent assistance with data collection.

Contributors RE and YKS (Yasuhiko Sakata) participated in the study concept and design. DN, SS, MU, SM and MH participated in the acquisition of the data. RE, YKS, SY and TH participated in the analysis and interpretation of the data. YKS, TK, HS, SH, YSS (Yasushi Sakata), SY, MH and TH participated in drafting and critical revision of the manuscript for important intellectual content. RE and TH participated in statistical analysis. YKS, HS, SN, MH and IK obtained funding.

Funding This work was supported by Grants-in-Aid for University and Society Collaboration (\#19590816 and \#19390215) from the Japanese Ministry of Education, Culture, Sports, Science and Technology, Tokyo, Japan.

Competing interests IK has received research grants and speaker's fees from Takeda Pharmaceutical Company, Astellas Pharma, Daiichi Sankyo Company, Nippon Boehringer Ingelheim, Novartis Pharma, Shionogi, and Kowa Pharmaceutical Company.

Patient consent Obtained.

Ethics approval The study protocol has been approved by the ethics committee of each participating hospital.
Provenance and peer review Not commissioned; externally peer reviewed.

Data sharing statement No additional data are available.

Open Access This is an Open Access article distributed in accordance with the Creative Commons Attribution Non Commercial (CC BY-NC 3.0) license, which permits others to distribute, remix, adapt, build upon this work noncommercially, and license their derivative works on different terms, provided the original work is properly cited and the use is non-commercial. See: http:// creativecommons.org/licenses/by-nc/3.0/

\section{REFERENCES}

1. Muller JE, Stone PH, Turi ZG, et al. Circadian variation in the frequency of onset of acute myocardial infarction. $N$ Engl J Med 1985;313:1315-22.

2. Mahmoud KD, Lennon $\mathrm{RJ}$, Ting $\mathrm{HH}$, et al. Circadian variation in coronary stent thrombosis. JACC Cardiovasc Interv 2011;4:183-90.

3. Muller JE, Tofler GH, Stone PH. Circadian variation and triggers of onset of acute cardiovascular disease. Circulation 1989;79:733-43.

4. Kinjo K, Sato H, Sato H, et al.; Osaka Acute Coronary Insufficiency Study (OACIS) Group. Circadian variation of the onset of acute myocardial infarction in the Osaka area, 1998-1999: characterization of morning and nighttime peaks. Jpn Circ J 2001;65:617-20.

5. Peters RW, Zoble RG, Liebson PR, et al. Identification of a secondary peak in myocardial infarction onset 11 to 12 hours after awakening: the Cardiac Arrhythmia Suppression Trial (CAST) experience. J Am Coll Cardiol 1993;22:998-1003.

6. Itaya $\mathrm{H}$, Takagi $\mathrm{T}$, Sugi $\mathrm{K}$, et al. Contents of second peak in the circadian variation of acute myocardial infarction in the Japanese population. J Cardiol 2012;59:147-53.

7. Tsukada $\mathrm{T}$, Ikeda $\mathrm{T}$, Ishiguro $\mathrm{H}$, et al. Circadian variation in out-of-hospital cardiac arrests due to cardiac cause in a Japanese patient population. Circ J 2010;74:1880-7.

8. Willich $\mathrm{SN}$, Löwel $\mathrm{H}$, Lewis $\mathrm{M}$, et al. Weekly variation of acute myocardial infarction. Increased Monday risk in the working population. Circulation 1994;90:87-93.

9. Hara M, Sakata Y, Nakatani D, et al. Osaka Acute Coronary Insufficiency Study (OACIS) Investigators. Low levels of serum n-3 polyunsaturated fatty acids are associated with worse heart failure-free survival in patients after acute myocardial infarction. Circ J 2013:1:153-62.

10. Nakatani D, Sakata Y, Suna S, et al. Osaka Acute Coronary Insufficiency Study (OACIS) Investigators. Impact of beta blockade therapy on long-term mortality after ST-segment elevation acute myocardial infarction in the percutaneous coronary intervention era Am J Cardiol 2013;111:457-64.

11. Gao F, Chia KS, Krantz I, et al. On the application of the von Mises distribution and angular regression methods to investigate the seasonality of disease onset. Stat Med 2006;25:1593-618.

12. Stergiou GS, Vemmos KN, Pliarchopoulou KM, et al. Parallel morning and evening surge in stroke onset, blood pressure, and physical activity. Stroke 2002;33:1480-6.

13. Degaute JP, van de Borne P, Linkowski $P$, et al. Quantitative analysis of the 24-hour blood pressure and heart rate patterns in young men. Hypertension 1991;18:199-210.

14. Kotsis V, Stabouli S, Bouldin M, et al. Impact of obesity on 24-hour ambulatory blood pressure and hypertension. Hypertension 2005;45:602-7.

15. Thrall G, Lane D, Carroll D, et al. A systematic review of the prothrombotic effects of an acute change in posture: a possible mechanism underlying the morning excess in cardiovascular events? Chest 2007;132:1337-47.

16. Tofler GH, Brezinski D, Schafer Al, et al. Concurrent morning increase in platelet aggregability and the risk of myocardial infarction and sudden cardiac death. N Engl J Med 1987;316:1514-18.

17. Andreotti F, Davies GJ, Hackett DR, et al. Major circadian fluctuations in fibrinolytic factors and possible relevance to time of onset of myocardial infarction, sudden cardiac death and stroke. Am J Cardiol 1988;62:635-7.

18. Tofler GH, Muller JE. Triggering of acute cardiovascular disease and potential preventive strategies. Circulation 2006;114:1863-72.

19. Hokanson JE, Austin MA. Plasma triglyceride level is a risk factor for cardiovascular disease independent of high-density lipoprotein cholesterol level: a meta-analysis of population-based prospective studies. J Cardiovasc Risk 1996;3:213-19.

20. Iso $\mathrm{H}$, Naito $\mathrm{Y}$, Sato $\mathrm{S}$, et al. Serum triglycerides and risk of coronary heart disease among Japanese men and women. Am J Epidemiol 2001;153:490-9. 
21. Masuda D, Sugimoto T, Tsujii K, et al. Correlation of fasting serum apolipoprotein B-48 with coronary artery disease prevalence. Eur J Clin Invest 2012;42:992-9.

22. Stampfer MJ, Krauss RM, Ma J, et al. A prospective study of triglyceride level, low-density lipoprotein particle diameter, and risk of myocardial infarction. JAMA 1996;276:882-8.

23. Nordestgaard BG, Benn M, Schnohr P, et al. Nonfasting triglycerides and risk of myocardial infarction, ischemic heart disease, and death in men and women. JAMA 2007;298:299-308.

24. No authors listed. Hypertriglyceridaemia and vascular risk. Report of a meeting of physicians and scientists, University College London Medical School. Lancet 1993;342:781-7.

25. Rosenson RS, Lowe GD. Effects of lipids and lipoproteins on thrombosis and rheology. Atherosclerosis 1998;140:271-80.

26. Hamsten A, Wiman B, de Faire U, et al. Increased plasma levels of a rapid inhibitor of tissue plasminogen activator in young survivors of myocardial infarction. N Engl J Med 1985;313:1557-63.

27. Mehta J, Mehta P, Lawson D, et al. Plasma tissue plasminogen activator inhibitor levels in coronary artery disease: correlation with age and serum triglyceride concentrations. J Am Coll Cardiol 1987;9:263-8

28. Eriksson P, Nilsson L, Karpe F, et al. Very-low-density lipoprotein response element in the promoter region of the human plasminogen activator inhibitor-1 gene implicated in the impaired fibrinolysis of hypertriglyceridemia. Arterioscler Thromb Vasc Biol 1998;18:20-6.

29. Mitropoulos KA, Miller GJ, Reeves BE, et al. Factor VII coagulant activity is strongly associated with the plasma concentration of large lipoprotein particles in middle-aged men. Atherosclerosis 1989;76:203-8.

30. Bruckert E, Carvalho de Sousa J, Giral P, et al. Interrelationship of plasma triglyceride and coagulant factor VII levels in normotriglyceridemic hypercholesterolemia. Atherosclerosis 1989;75:129-34

31. Rosenson RS, Shott S, Tangney CC. Hypertriglyceridemia is associated with an elevated blood viscosity Rosenson: triglycerides and blood viscosity. Atherosclerosis 2002;161:433-9.

32. Hamsten A, de Faire U, Walldius $G$, et al. Plasminogen activator inhibitor in plasma: risk factor for recurrent myocardial infarction. Lancet 1987;2:3-9.
33. Meade TW, Mellows S, Brozovic M, et al. Haemostatic function and ischaemic heart disease: principal results of the Northwick Park Heart Study. Lancet 1986;2:533-7.

34. Lowe GD, Lee AJ, Rumley A, et al. Blood viscosity and risk of cardiovascular events: the Edinburgh Artery Study. Br J Haematol 1997;96:168-73.

35. Lundman P, Eriksson M, Schenck-Gustafsson K, et al. Transient triglyceridemia decreases vascular reactivity in young, healthy men without risk factors for coronary heart disease. Circulation 1997;96:3266-8.

36. Kugiyama K, Doi H, Motoyama $\mathrm{T}$, et al. Association of remnant lipoprotein levels with impairment of endothelium-dependent vasomotor function in human coronary arteries. Circulation 1998:97:2519-26.

37. Zeiher AM, Drexler H, Saurbier B, et al. Endothelium-mediated coronary blood flow modulation in humans. Effects of age, atherosclerosis, hypercholesterolemia, and hypertension. J Clin Invest 1993;92:652-62.

38. Rivera-Coll A, Fuentes-Arderiu X, Díez-Noguera A. Circadian rhythmic variations in serum concentrations of clinically important lipids. Clin Chem 1994;40:1549-53.

39. Kario K, Yano Y, Matsuo T, et al. Additional impact of morning haemostatic risk factors and morning blood pressure surge on stroke risk in older Japanese hypertensive patients. Eur Heart $J$ 2011;32:574-80.

40. Richards J, Gumz ML. Advances in understanding the peripheral circadian clocks. FASEB J 2012;26:3602-13.

41. Kohsaka A, Waki $\mathrm{H}$, Cui $\mathrm{H}$, et al. Integration of metabolic and cardiovascular diurnal rhythms by circadian clock. Endocr $J$ 2012;59:447-56.

42. Manfredini R, Boari B, Bressan S, et al. Influence of circadian rhythm on mortality after myocardial infarction: data from a prospective cohor of emergency calls. Am J Emerg Med 2004;22:555-9.

43. Bae $\mathrm{MH}$, Ryu HM, Lee $\mathrm{JH}$, et al. The impact of circadian variation on 12-month mortality in patients with acute myocardial infarction. Korean Circ J 2010;40:616-24.

44. Holmes DR Jr, Aguirre FV, Aplin R, et al. Circadian rhythms in patients with ST-elevation myocardial infarction. Circ Cardiovasc Qual Outcomes 2010;3:382-9. 